\section{Definitive endoscopic pyloric exclusion with an over-the-scope clip}

A 48-year-old man underwent primary surgical repair of an iatrogenic duodenal perforation, sustained during endoscopic ultrasonography. After 2 weeks, dehiscence of the suture with intra-abdominal infection and abscesses was identified, for which pyloric-duodenal dissociation with pyloric closure, duodenostomy, gastrojejunostomy, and multiple drainages were performed. Spontaneous opening of the sutured pylorus with leakage of contrast was observed 4 weeks later.

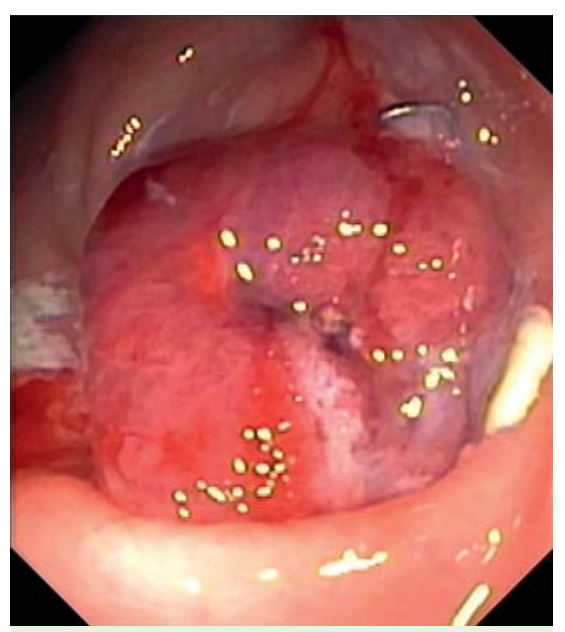

Fig. 1 In a 48-year-old man undergoing endoscopic closure of the pylorus to manage infection and dehiscence of a previously sutured iatrogenic duodenal perforation, an endoscopic image shows the over-the-scope clip (OTSC) immediately after its release into the pylorus. The pylorus was previously inverted into the cap of the OTSC. The OTSC completely entraps the pylorus.

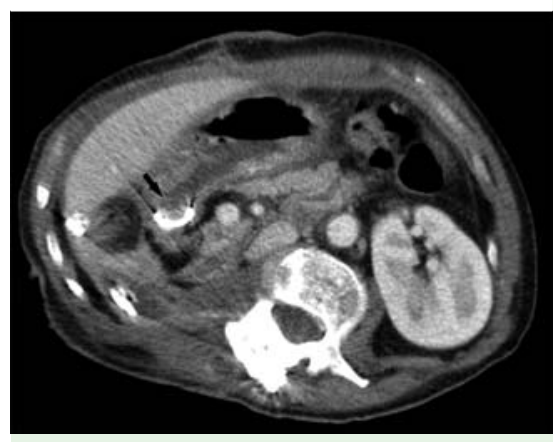

Fig. 2 Computed tomographic scan obtained 3 days after endoscopic pyloric exclusion.

The over-the-scope clip is seen entrapping the pylorus (arrow).
Upon endoscopic evaluation, the gastrojejunal anastomosis was normal, the pylorus was completely open, and the perforation, extruding abundant purulent content, was still identified. To avoid a third surgery, endoscopic closure of the pylorus was planned. Initially, argon plasma coagulation (APC) was used to denude the duodenal side of the pylorus. Then, a 14-t over-the-scope-clip (OTSC; Ovesco Endoscopy AG, Tübingen, Germany) was applied to the endoscope, and suction was used to invert the pylorus into the cap ( $\bullet$ Video 1 ). With the pylorus in that position, the clip was released, resulting in circular entrapment of the inverted pylorus. Correct positioning of the clip around the inverted pylorus was confirmed ( Fig. 1 and $\bullet$ Fig.2). APC was then applied on the gastric side of the pylorus.

After the patient had ingested $200 \mathrm{~mL}$ of a methylene blue solution the next day, the absence of extravasation from the drains was confirmed, and oral intake was resumed. Clinical and radiologic improvement was seen in the following weeks, and the patient was discharged 4 weeks after the endoscopic procedure. Upon reevaluation 9 months later, the OTSC was observed in the expected position, with the inverted pylorus completely entrapped and closed by the clip ( $\bullet$ Figs.3-5).

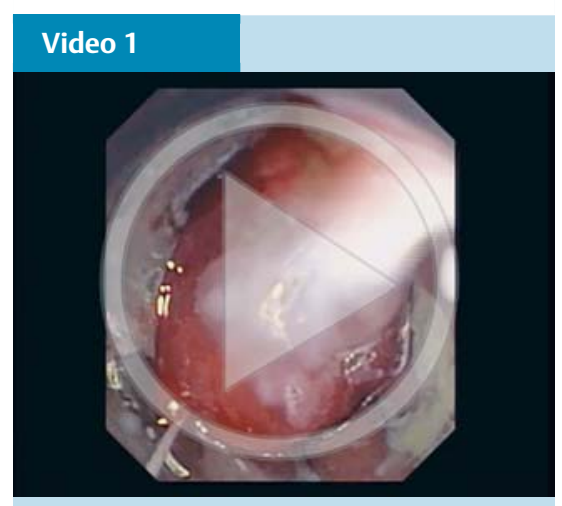

Endoscopic and computed tomographic video showing suction of the pylorus and release of the over-the-scope clip with complete entrapment of the pylorus inside the clip, argon plasma coagulation of the pylorus, and endoscopic confirmation of definitive pyloric exclusion 9 months later.
The OTSC has been used successfully to close digestive perforations, anastomotic leaks, fistulas, and gastrostomies after natural orifice transluminal endoscopic surgery. It has also been used to treat complications of bariatric surgery and bleeding lesions, to resect submucosal tumors, and to fix stents [1-5]. Herein,

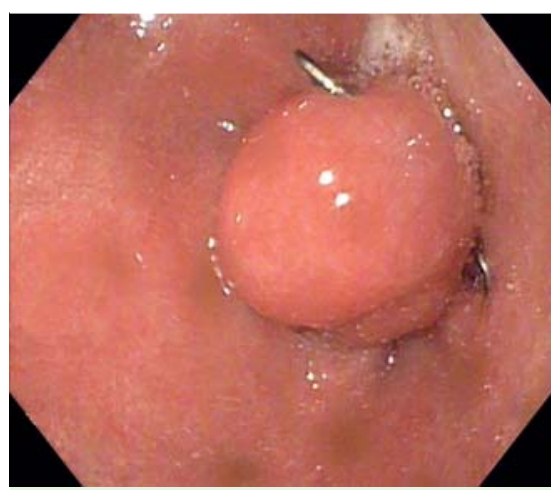

Fig. 3 Endoscopic image obtained 9 months after endoscopic exclusion of the pylorus shows the pylorus entrapped and circularly closed by the OTSC.

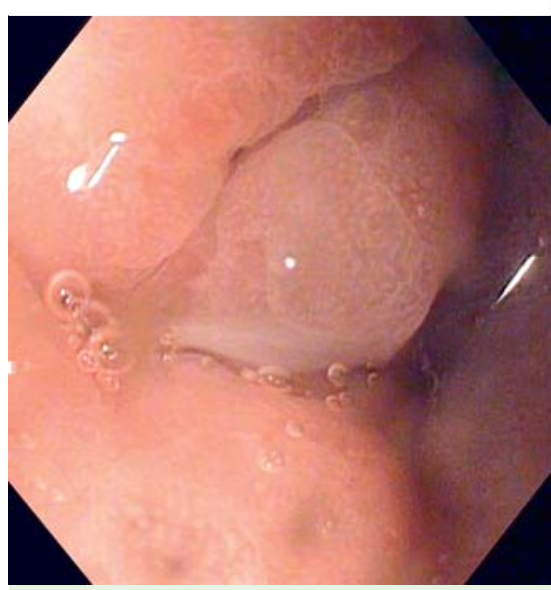

Fig. 4 Same as Fig. 3, with a focus on the previous pyloric opening, which is now closed and epithelialized.

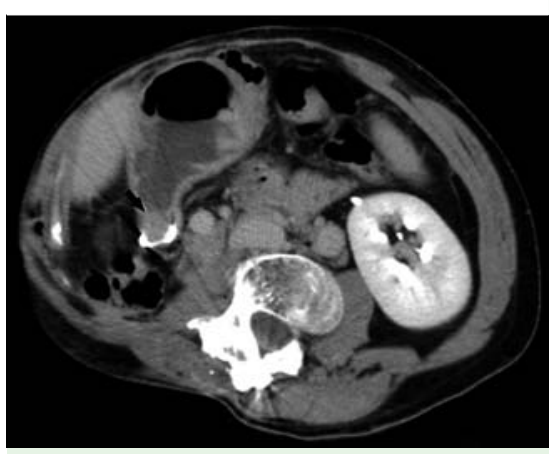

Fig. 5 Computed tomographic scan obtained 9 months after endoscopic pyloric exclusion. The over-the-scope clip is seen entrapping the pylorus (arrow). 
the authors present another indication that to the best of their knowledge has not previously been reported - definitive endoscopic pyloric exclusion after dehiscence of surgical pyloric exclusion.

Endoscopy_UCTN_Code_TTT_1AO_2AI

Competing interests: None

\section{Rolando Pinho' ${ }^{1}$, Carlos Fernandes', Alexandre Costa', Luísa Proença', Sónia Fernandes ${ }^{1}$, Jorge Carrapita ${ }^{2}$, João Carvalho ${ }^{1}$}

${ }^{1}$ Gastroenterology Department, Centro Hospitalar de Vila Nova de Gaia, Portugal 2 Surgery Department, Centro Hospitalar de Vila Nova de Gaia, Portugal

\section{References}

1 Seebach L, Bauerfeind P, Gubler C. "Sparing the surgeon": clinical experience with overthe-scope clips for gastrointestinal perforation. Endoscopy 2010; 42: 1108-1111

2 Gubler C, Bauerfeind P. Endoscopic closure of iatrogenic gastrointestinal tract perforations with the over-the-scope clip. Digestion 2012; 85: 302-307

3 Sandmann M, Heike M, Faehndrich M. Application of the OTSC system for the closure of fistulas, anastomosal leakages and perforations within the gastrointestinal tract. Z Gastroenterol 2011; 49: 981-985

4 Mudumbi S, Velazquez-Aviña J, Neumann $H$ et al. Anchoring of self-expanding metal stents using the over-the-scope clip, and a technique for subsequent removal. Endoscopy 2014; 46: 1106-1109

5 Mönkemüller K, Peter S, Toshniwal J et al. Multipurpose use of the 'bear claw' (overthe-scope-clip system) to treat endoluminal gastrointestinal disorders. Dig Endosc 2014; 26: $350-357$
Bibliography

DoI http://dx.doi.org/

10.1055/s-0034-1392260

Endoscopy 2015; 47: E323-E324

(c) Georg Thieme Verlag KG

Stuttgart · New York

ISSN 0013-726X

\section{Corresponding author}

\section{Rolando Pinho, MD}

Serviço de Gastrenterologia

Centro Hospitalar de Vila Nova de Gaia

Rua Conceição Fernandes

4434-502 Vila Nova de Gaia

Portugal

Fax: +351-22-786-8369

rolandopinho@gmail.com 